\title{
Outage Probability and Energy Efficiency of DF Relaying Power Line Communication Networks: Cooperative and Non-Cooperative
}

\author{
Khaled M. Rabie*, Bamidele Adebisi* and Haris Gacanin ${ }^{\ddagger}$ \\ ${ }^{*}$ School of Electrical Engineering, Manchester Metropolitan University, Manchester, UK. \\ ${ }_{\ddagger}^{\ddagger}$ Nokia, Copernicuslaan 50, 2018 Antwerp, Belgium \\ Email: \{k.rabie@mmu.ac.uk; b.adebisi@mmu.ac.uk; haris.gacanin@nokia.com\}
}

\begin{abstract}
This paper analyzes the energy efficiency performance of cooperative and non-cooperative decode-and-forward (DF) relaying power line communication (PLC) systems. In order to further minimize the energy consumption of such systems, we propose incremental DF (IDF) relying over the impulsive noise PLC channel. For a more realistic scenario, the PLC modems power consumption profile is assumed to consist of both dynamic power and static power. For the sake of comparison and completeness as well as to quantify the achievable gains, we also analyze the performance of a single-hop PLC system. In this respect, accurate analytical expressions for the outage probability and energy efficiency are derived. Monte Carlo simulations are provided throughout the paper to validate the analysis. Results reveal that the cooperative relaying PLC systems can provide better energy efficiency performance compared to the non-cooperative ones. It is also shown that increasing the noise probability or the modems static power can negatively impact the system performance.
\end{abstract}

Index Terms-Impulsive noise, incremental DF, log-normal fading, selective DF, outage probability, power line communication (PLC).

\section{INTRODUCTION}

A DVANCES in modulation schemes and signal processing algorithms have made reliable and high data-rate communication over power line networks possible, despite their unfavorable physical properties at high frequencies [1]-[4]. This technology is commonly referred to as power line communications (PLCs). The main advantage of PLC resides in the significant reduction of deployment costs and the elimination of hard-to-reach access problem in buildings, e.g., cellars. Similar to many originally-designed wireless technologies, cooperative relaying systems including amplify-and-forward (AF) and decode-and-forward (DF) have recently found their way to the PLC domain [5]-[7]. It was shown that remarkable capacity improvements can be attained with the use of relays and that the PLC system reliability can be further improved by increasing the number of relaying modems in comparison to the direct-link transmission. However, increasing the number of relays increases the total power consumption due to the static power of each modem.

In recent years, a number of studies have appeared in the literature investigating the issue of power consumption and energy efficiency of relaying PLC systems [8]-[10]. Follow- ing this considerable research attention devoted to energy efficiency in PLC in recent times, it is set to become a critical design criterion in future PLC networks. These studies considered different relaying and power consumption profiles of PLC modems to reduce transmit power and enhance energy efficiency. This is mainly achieved by optimizing system parameters. On the contrary, the authors in [11], [12] have proposed harvesting the unwanted high impulsive noise energy which is mostly wasted due to the blanking/clipping process at the receiving modem [13]-[15].

To the best of our knowledge, all the aforementioned works are based on experimental measurements and no in-depth analytical analysis of the energy efficiency in relaying PLC systems is performed. This paper is therefore dedicated to analyze the outage probability and energy efficiency performance of four non-cooperative and cooperative DF PLC systems. More specifically, we consider two non-cooperative transmission strategies, namely, single-hop and non-cooperative DF relaying (also referred to conventional DF relaying), and two cooperative schemes, namely, selective DF (SDF) and incremental DF (IDF). The main contribution of this paper resides in deriving accurate analytical expressions for the average outage probability and energy efficiency (in terms of the energy-per-bit) of the four non-cooperative and cooperative systems under consideration. Further, we provide insightful comparisons between these systems in several environments. The results reveal that cooperative transmission, both SDF and IDF protocols, can considerably improve the outage probability performance. It is also demonstrated that the IDF PLC approach is able to offer the best energy efficiency followed by the SDF scheme. Further, increasing the end-toend distance, impulsive noise probability, the channel variance or the static power of PLC modems will remarkably deteriorate the performance of all the four systems.

The rest of this paper is organized as follows. Section II presents the system model. In Section III, we analyze the average outage probability and energy-per-bit performance of the single-hop, conventional DF, SDF and IDF relaying systems. Numerical examples and simulation results are presented and discussed in Section IV. Finally, Section V concludes the paper. 


\section{SySTEM MODEL}

The main system model considered in this study consists of a source modem $(\mathrm{S})$, relaying modem $(\mathrm{R})$ and destination modem (D). The source-to-relay, relay-to-destination and sourceto-destination channel coefficients follow log-normal distribution and are denoted as $h_{1}, h_{2}$ and $h_{0}$, with the corresponding distances being $d_{1}, d_{2}$ and $d_{0}=d_{1}+d_{2}$, respectively. The means and standard deviations of $10 \log _{10}\left(h_{m}\right)$ are denoted as $\mu_{h_{m}}$ and $\sigma_{h_{m}}$, respectively, where $m \in\{0,1,2\}$. In addition, the cable losses are modeled as $A\left(f, d_{m}\right)=\exp \left(-\alpha d_{m}\right)$, where $\alpha=a_{o}+a_{1} f^{k}$ is the attenuation factor, $f$ is the operating frequency, $k$ is the exponent of the attenuation factor, $d_{m}$ is the distance, and $a_{o}$ and $a_{1}$ are constants determined from measurements which depend on the network topology and structure [1].

Four transmission strategies are studied, two of which are non-cooperative and the other two are cooperative. The noncooperative ones are single-hop and conventional DF whereas the cooperative schemes are SDF and IDF. The energy efficiency in this study is calculated as the transmit energy-per-bit, which is basically the total transmit power over the data rate. The adopted power consumption profile takes into account not only the dynamic power but also the static power of the PLC modems consumed by the circuitry [9], [16].

Furthermore, to accurately characterize the PLC channel impairments, the noise at all modems is assumed to consist of both background noise $\left(n_{w}\right)$ with variance $\sigma_{w}^{2}$ and impulsive noise $\left(n_{i}\right)$ with variance $\sigma_{i}^{2}$. The Gaussian-Bernoulli noise model is used to characterize these noise components and the probability occurrence of impulsive noise is represented by $p$ [17]. The variances $\sigma_{w}^{2}$ and $\sigma_{i}^{2}$ define the signal-tobackground noise ratio (SBNR) and the signal-to-impulsive noise ratio $(\mathrm{SINR})$, respectively, as $\mathrm{SBNR}=10 \log _{10}\left(1 / \sigma_{w}^{2}\right)$ and $\operatorname{SINR}=10 \log _{10}\left(1 / \sigma_{i}^{2}\right)$.

\section{Performance Analysis}

In this section, we analyze the average outage probability and energy efficiency performances of the four transmission schemes above.

\section{A. Non-cooperative Transmission}

Single-hop and conventional DF relaying are two noncooperative transmission schemes analyzed in this section. In the single-hop approach, the source communicates directly with the destination modem whereas in the conventional DF relaying system, the end-to-end communication is accomplished by the help of an intermediate relaying modem.

1) Single-hop Transmission: the energy-per-bit of the single-hop scheme is given by

$$
E_{b, \mathrm{SH}}=\frac{P_{\mathrm{SH}}^{*}+P_{s t c}^{T x}+P_{s t c}^{R x}}{R_{b}}
$$

where $E_{b}$ is the energy/bit, $P_{\mathrm{SH}}^{*}$ is the optimal transmit power for a given outage probability, i.e. the minimum transmit power to achieve a certain outage probability, $R_{b}=\xi B$ is the data rate in bits/s, $\xi$ is the spectral efficiency and $B$ is the system bandwidth, in $\mathrm{Hz}, P_{s t c}^{T x}$ and $P_{s t c}^{R x}$ are the static powers of the transmitting and receiving modems, respectively. It should be highlighted that in order to minimize the overall energy consumption, the transmit power must be minimized since the static power is circuitry-specific.

We now derive the outage probability for this system. To begin with, the received signal at the destination modem in the case of single-hop transmission can be written as

$$
y_{D}=P_{\mathrm{SH}} A\left(f, d_{0}\right) h_{0}^{2} x+n_{w}+n_{i}
$$

where $P_{\mathrm{SH}}$ is the source transmit power and $x$ is the source information signal.

The signal-to-background noise ratio (SBNR) at the destination can then be expressed as

$$
\gamma_{D}=\frac{P_{\mathrm{SH}} A\left(f, d_{0}\right) h_{0}^{2}}{\sigma_{w}^{2}} .
$$

In impulsive noise channels, such as the power line channel, the outage probability $P_{\text {out }}^{\mathrm{SD}}$ can be determined as follows [18], [19]

$$
P_{\mathrm{out}}^{\mathrm{SD}}=\operatorname{Pr}\left\{\sum_{i=0}^{1} p_{i} \log _{2}\left(1+\gamma_{D, i}\right)<\xi\right\}
$$

where $\gamma_{D, 0}=\gamma_{D}, \gamma_{D, 1}=\gamma_{D} / \beta$ and $\beta=1+\sigma_{i}^{2} / \sigma_{w}^{2}$.

To simplify our analysis, we use the high signal-to-noise ratio (SNR) approximation [20]. Therefore, (4) can be reduced to

$$
P_{\mathrm{out}}^{\mathrm{SD}} \simeq \operatorname{Pr}\left\{\left(\gamma_{D}\right)^{1-p}\left(\frac{\gamma_{D}}{\beta}\right)^{p}<2^{\xi}\right\}
$$

which can also be be written as

$$
P_{\text {out }}^{\mathrm{SD}} \simeq \operatorname{Pr}\left\{\gamma_{D}<\beta^{p} 2^{\xi}\right\}
$$

It is clear that the expression in (6) basically represents the cumulative distribution function (CDF) of $\gamma_{D}$. Hence, (6) can be expressed as

$$
P_{\mathrm{out}}^{\mathrm{SD}} \simeq F_{\gamma_{D}}\left(\beta^{p} 2^{\xi}\right)
$$

where $F_{\gamma_{D}}(\cdot)$ is the CDF of $\gamma_{D}$.

From (3), clearly $\gamma_{D}$ has log-normal distribution because $h_{0}^{2}$ is log-normally distributed. Consequently, and using the properties of log-normal distribution, the outage probability $P_{\text {out }}^{\text {SD }}$ can be now written as

$$
P_{\text {out }}^{\mathrm{SD}} \simeq \frac{1}{2}+\frac{1}{2} \operatorname{erf}\left(\frac{\zeta \ln \left(\beta^{p} 2^{\xi}\right)-\left(2 \mu_{h_{0}}+\zeta \ln \left[\Xi_{0}\right]\right)}{\sqrt{8} \sigma_{h_{0}}}\right),
$$

where $\zeta=10 / \ln (10)$ is a scaling constant and $\Xi_{0}=$ $P_{\mathrm{SH}} A\left(f, d_{0}\right) / \sigma_{w}^{2}$. 
Now, fixing the outage probability at the limit $P_{\text {out }}^{*}$ and using (8), we can determine the optimal transmit power as

$$
P_{\mathrm{SH}}^{*}=\frac{\beta^{p} 2^{\xi} \sigma_{w}^{2}}{A\left(f, d_{0}\right)} \exp \left(-\frac{\sqrt{8} \sigma_{h_{0}} \operatorname{erf}^{-1}\left(2 P_{\mathrm{out}}^{*}-1\right)+2 \mu_{h_{0}}}{\zeta}\right) .
$$

Finally, substituting (9) into (1) yields the energy-per-bit performance of the single-hop PLC system.

2) Conventional DF Relaying: we analyze in this section the energy efficiency of a non-cooperative dual-hop PLC system with DF relaying. DF relaying was selected because it was recently shown to have better performance over the impulsive noise PLC channel compared to the AF protocol. The total energy-per-bit consumption of this system is calculated as

$$
E_{b, \mathrm{DF}}=\frac{P_{\mathrm{DF}}^{*}+P_{s t c}^{T x}+P_{s t c}^{R x}}{N R_{b}}\left(P_{\mathrm{out}}^{\mathrm{SR}}+2\left(1-P_{\mathrm{out}}^{\mathrm{SR}}\right)\right),
$$

where $P_{\text {out }}^{\mathrm{SR}}$ denotes the outage probability of the sourceto-relay link and $P_{\mathrm{DF}}^{*}$ is the optimal transmit power for a given outage probability of the overall system. Note that a parameter $N$, which is equal to 2 in this case, appeared in the denominator. This is because of the fact that in the dualhop relaying system, the overall throughput will be reduced to half since the end-to-end communication is now accomplished over two time slots. Note that the first and second terms in (10) represent the energy consumption when the decoding at the relay is unsuccessful and successful, respectively.

To calculate the energy efficiency of this system, we first need to derive the overall outage probability $\left(P_{\text {out }}^{\mathrm{DF}}\right)$ which will then allow us to determine the optimal transmit power $\left(P_{\mathrm{DF}}^{*}\right)$. Assuming that the relay is placed midway between the source and destination, the end-to-end outage probability of this system can be given by

$$
P_{\text {out }}^{\mathrm{DF}}=P_{\text {out }}^{\mathrm{SR}}+\left(1-P_{\text {out }}^{\mathrm{SR}}\right) P_{\text {out }}^{\mathrm{RD}},
$$

where $P_{\text {out }}^{\mathrm{SR}}$ and $P_{\mathrm{out}}^{\mathrm{RD}}$ are the outage probabilities of the sourceto-relay and relay-to-destination links, respectively.

Similar to the derivation in the previous section, and assuming that the source and relay have same transmit power of $P_{\mathrm{DF}}$, the outage probabilities $P_{\mathrm{out}}^{\mathrm{SR}}$ and $P_{\text {out }}^{\mathrm{RD}}$ can be respectively expressed as

$$
\begin{aligned}
& P_{\text {out }}^{\mathrm{SR}} \simeq \frac{1}{2}+\frac{1}{2} \operatorname{erf}\left(\frac{\zeta \ln \left(\beta^{p} 2^{\xi}\right)-\left(2 \mu_{h_{1}}+\zeta \ln \left[\Xi_{1}\right]\right)}{\sqrt{8} \sigma_{h_{1}}}\right), \\
& P_{\text {out }}^{\mathrm{RD}} \simeq \frac{1}{2}+\frac{1}{2} \operatorname{erf}\left(\frac{\zeta \ln \left(\beta^{p} 2^{\xi}\right)-\left(2 \mu_{h_{2}}+\zeta \ln \left[\Xi_{2}\right]\right)}{\sqrt{8} \sigma_{h_{2}}}\right),
\end{aligned}
$$

where $\Xi_{1}=P_{\mathrm{DF}} A\left(f, d_{1}\right) / \sigma_{w}^{2}$ and $\Xi_{2}=P_{\mathrm{DF}} A\left(f, d_{2}\right) / \sigma_{w}^{2}$.

Now, substituting (12) and (13) into (11) while replacing $P_{\text {out }}^{\mathrm{DF}}$ with $P_{\text {out }}^{*}$, we can numerically find the optimal transmit power $\left(P_{\mathrm{DF}}^{*}\right)$ for a given $P_{\mathrm{out}}^{*}$. Finally, we substitute the obtained $P_{\mathrm{DF}}^{*}$ into (10) to yield the consumed energy-per-bit for the non-cooperative DF system.

\section{B. Cooperative Transmission}

In cooperative transmission, the source transmits its information signal to the destination during the first time slot which can also be heard by the relaying modem. In the second time slot, the relay will decode and forward the received signal to the destination. The destination then performs selection combining based on the two received copies from the source and relay modems during the two time slots. On one hand, the relay in the SDF scheme will always cooperate as long as it successfully decodes the received signal. On the other hand, the relay in the IDF protocol will only cooperate if requested to do so by the destination modem. To achieve this, a feedback channel is assumed to exist between the relay and destination in the IDF based system.

1) Selective DF PLC System: the total consumed energyper-bit in this system is given by

$$
\begin{aligned}
E_{b, \mathrm{SDF}}= & P_{\mathrm{out}}^{\mathrm{SR}} \frac{P_{\mathrm{SDF}}^{*}+P_{s t c}^{T x}+2 P_{s t c}^{R x}}{N R_{b}} \\
& +\left(1-P_{\mathrm{out}}^{\mathrm{SR}}\right) \frac{2 P_{\mathrm{SDF}}^{*}+2 P_{s t c}^{T x}+3 P_{s t c}^{R x}}{N R_{b}}
\end{aligned}
$$

where $P_{\mathrm{SDF}}^{*}$ is the optimal transmit power in the SDF PLC scheme for a given overall system outage probability which can be calculated for this case as

$$
P_{\text {out }}^{\mathrm{SDF}}=P_{\text {out }}^{\mathrm{SD}}\left(P_{\text {out }}^{\mathrm{SR}}+\left(1-P_{\text {out }}^{\mathrm{SR}}\right) P_{\text {out }}^{\mathrm{RD}}\right) .
$$

Note that the additional $P_{\text {stc }}^{R x}$ in (14) is due to the fact that the destination will be decoding the source signal at the same time as the relay, during the first time. The terms $P_{\text {out }}^{\mathrm{SD}}, P_{\mathrm{out}}^{\mathrm{SR}}$ and $P_{\mathrm{out}}^{\mathrm{SD}}$ in (15) can be obtained from (8), (12) and (13), respectively, by replacing $P_{\mathrm{SH}}$ and $P_{\mathrm{DF}}$ with $P_{\mathrm{SDF}}$. Then, replacing $P_{\text {out }}^{\mathrm{SDF}}$ in (15) with $P_{\text {out }}^{*}$, we can numerically find the corresponding optimal transmit power $P_{\mathrm{SDF}}^{*}$ which is then substituted into (14) to obtain the overall energy-per-bit performance of the SDF PLC system.

2) Incremental DF PLC System: the availability of the feedback channel in this protocol allows the relay to avoid unnecessary data transmission; hence, consuming less power and considerably improving the energy efficiency. This protocol is based on the fact that relaying will only take place when the direct-link is unable to provide sufficient link quality [21]. Under this assumption, the energy consumed per bit in this system can be written as

$$
\begin{aligned}
E_{b, \mathrm{IDF}}= & \left(1-P_{\mathrm{out}}^{\mathrm{SD}}\right) \frac{P_{\mathrm{IDF}}^{*}+P_{s t c}^{T x}+2 P_{s t c}^{R x}}{L R_{b}} \\
& +P_{\text {out }}^{\mathrm{SD}} P_{\text {out }}^{\mathrm{SR}} \frac{P_{\mathrm{IDF}}^{*}+P_{s t c}^{T x}+2 P_{s t c}^{R x}}{R_{b}} \\
& +P_{\text {out }}^{\mathrm{SD}}\left(1-P_{\text {out }}^{\mathrm{SR}}\right) \frac{2 P_{\mathrm{IDF}}^{*}+2 P_{s t c}^{T x}+3 P_{s t c}^{R x}}{L R_{b}}
\end{aligned}
$$

where $P_{\mathrm{IDF}}^{*}$ is the optimal transmit power of the IDF system for a given value of the end-to-end outage probability.

The outage probability of the IDF scheme is equal to that of the SDF system; therefore, it can be easily obtained from (15) 
by making the appropriate notation changes. Following the same procedure as in the case of SDF, it is straightforward to find the energy-per-bit for the IDF PLC system. This is omitted here for brevity.

\section{NuMERICAL RESUlts}

In this section, we present and discuss some numerical examples of the outage probability and energy efficiency expressions derived above. Throughout this section, if not specified, we use $a_{0}=9.4 \times 10^{-3}, a_{1}=4.2 \times 10^{-7}$, $k=0.7, f=30 \mathrm{MHz}, \mathrm{SBNR}=25 \mathrm{~dB}, \mathrm{SINR}=-15 \mathrm{~dB}$, $p=0.01, P_{\text {out }}^{*}=10^{-2}, \mu_{m}=4.5 \mathrm{~dB}, \sigma_{m}^{2}=2.5 \mathrm{~dB}$, where $m \in\{0,1,2\}$. In addition, we assume that the relaying PLC modem is always placed at the midpoint between the end modems.

\section{A. Outage Probability Performance}

The analytical and simulated outage probability results for the non-cooperative and cooperative DF PLC systems are shown in Fig. 1. The good agreement between the two results clearly indicates the accuracy of our analysis. The analytical results on this figure are obtained from (8), (11) and (15). It is evident from this figure that the two cooperative schemes, SDF and IDF, always outperform the non-cooperative systems and that the single-hop transmission has the poorest performance. It is also clear that SDF and IDF have identical outage performance since the same equation was used to obtain the outage probability for the two systems as discussed in the previous section. In addition, it is noticeable that, irrespective of the system deployed, the performance will degrade as the source and destination modems become more distant. It is worth pointing out that when the distance is extremely large, i.e., in this case greater than $2000 \mathrm{~m}$, even the performance of the cooperative schemes degrades significantly. To reduce the severity of this, and since the transmit power over power lines is restricted, more relaying modems will need to be deployed.

Fig. 2 depicts 3D plots for the outage probability with respect to the channel variance and end-to-end distance for the four non-cooperative and cooperative systems. It can be seen that increasing the channel variance and/or the sourceto-destination distance will deteriorate the system performance for all the systems considered. Comparing the results in Fig. 2(a) and Fig. 2(b), it is clear that the cooperative SDF and IDF systems always have remarkably better performance in comparison to the conventional DF approach for all the considered channel variances and distances.

\section{B. Energy-Per-Bit Performance}

This section presents the impact of various system parameters on the energy efficiency of the four schemes. Fig. 3 shows the energy-per-bit performance with respect to the endto-end distance for the single-hop, non-cooperative relaying, cooperative SDF and IDF systems with different impulsive noise probabilities and static power values; more specifically, $\left\{p=0.01\right.$ and $\left.P_{\text {stc }}=0.6 \mathrm{~W}\right\},\left\{p=0.01\right.$ and $\left.P_{\text {stc }}=3 \mathrm{~W}\right\}$ and $\left\{p=0.2\right.$ and $\left.P_{\text {stc }}=0.6 \mathrm{~W}\right\}$.

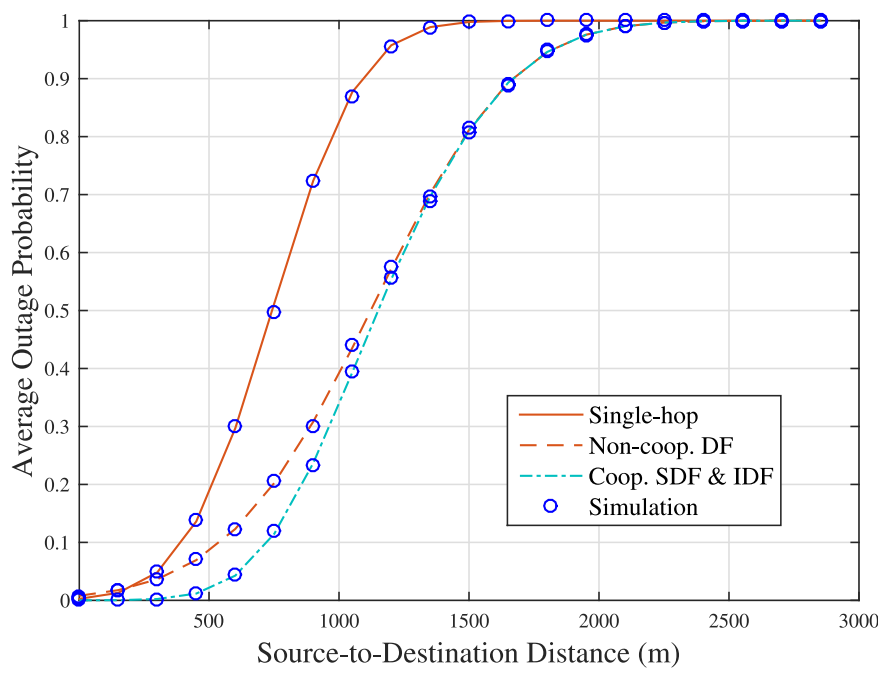

Figure 1: Average outage probability performance of the single-hop, noncooperative DF, SDF and IDF relaying systems as a function of the source-to-destination distance.

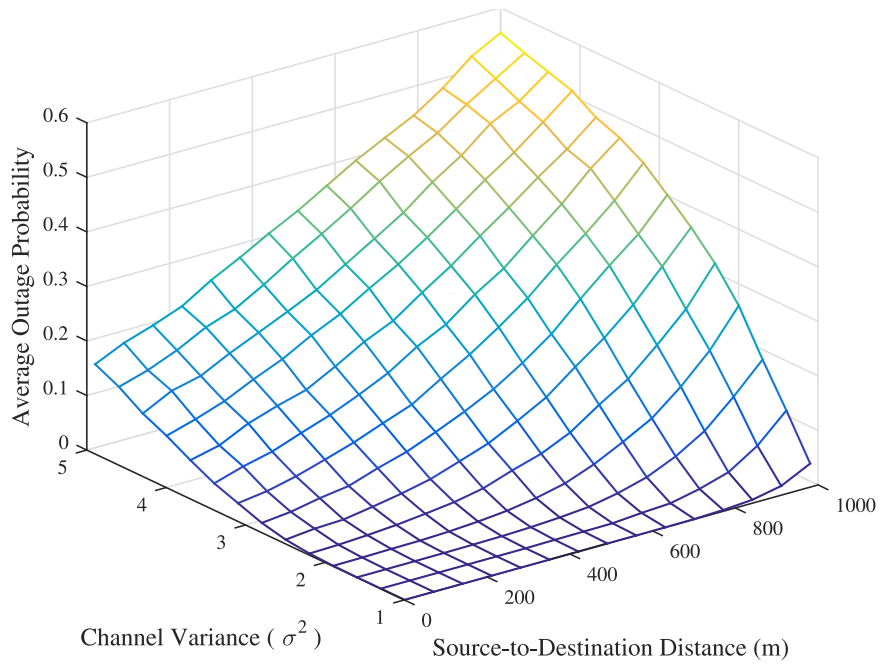

(a) Non-cooperative DF relaying.

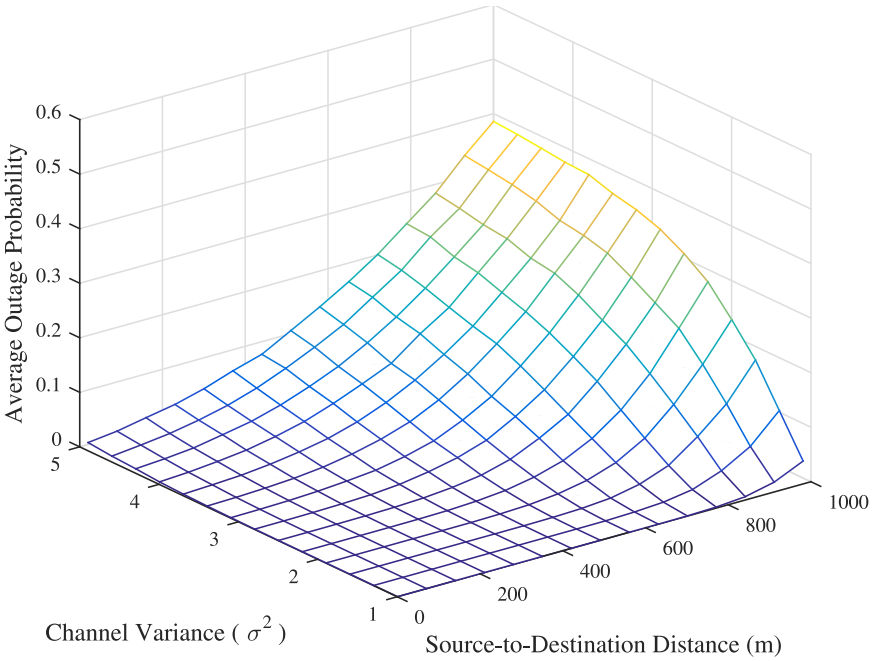

(b) Cooperative SDF and IDF relaying.

Figure 2: 3D plots for the average outage probability performance of the non-cooperative and cooperative DF systems as a function of the channel variance and source-to-destination distance. 


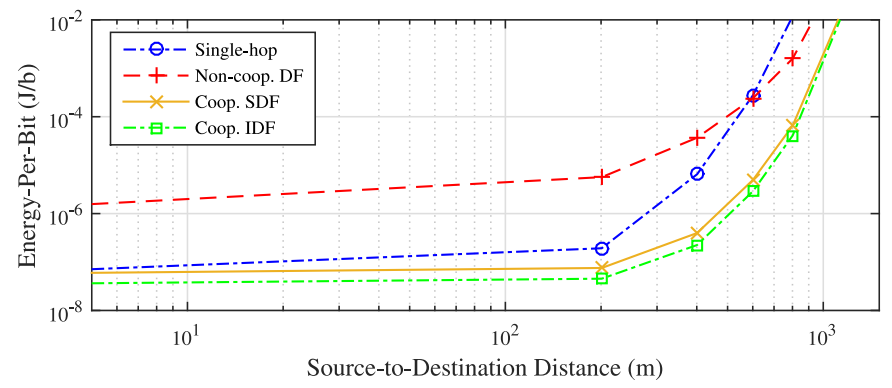

(a) $p=0.01$ and $P_{s t c}=0.6 \mathrm{~W}$

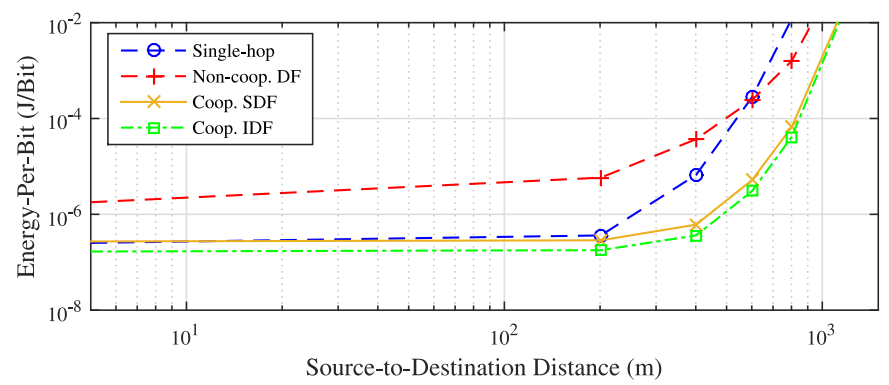

(b) $p=0.01$ and $P_{s t c}=3 \mathrm{~W}$

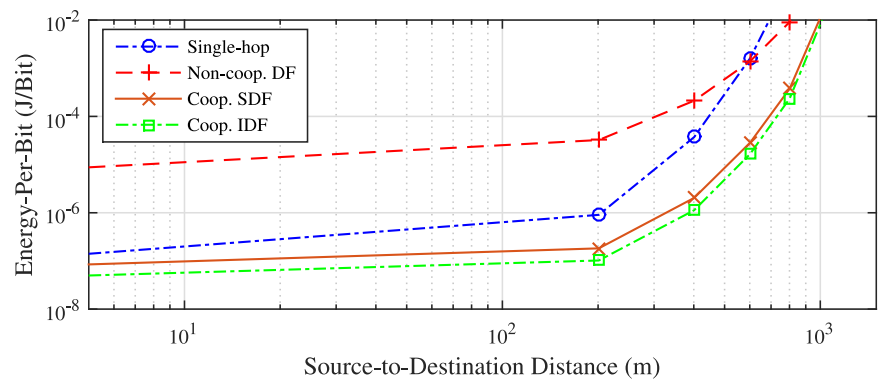

(c) $p=0.2$ and $P_{\text {stc }}=0.6 \mathrm{~W}$

Figure 3: Energy-per-bit performance with respect to end-to-end distance for the single-hop, conventional DF, SDF and IDF systems with various static power and impulsive noise configurations.

Comparing the results on this figure, it can be clearly noticed that the cooperative systems are more energy efficiency with IDF being the most efficient followed by SDF. This is always valid irrespective of the system configuration implemented. Having a closer look at these results, one can see that increasing the impulsive noise probability and/or the static power of the PLC modems will remarkably reduce the energy efficiency. Furthermore, interestingly enough, when the source-to-destination distance is relatively small, the single-hop approach tends to achieve better energy-per-bit performance in comparison to the non-cooperative DF system. This implies that at short distances the use of relays can be energy-inefficient due to the increased static power of the PLC modems. In contract, as the distance becomes larger, the advantage of using relays becomes clearer and non-cooperative DF outperforms the single-hop transmission.

\section{CONCLUSIONS}

In this paper, we examined the performance of four noncooperative and cooperative relaying PLC systems with lognormal fading and non-Gaussian noise. Specifically, single- hop, conventional DF, SDF and IDF transmission strategies were considered. The system performance of those protocols were analyzed in terms of the average outage probability and energy-per-bit performance. Simulation results clearly verified the correctness of the analysis. Results showed that cooperative transmission can offer improved outage probability and energy efficiency performance with the IDF PLC approach achieving the best performance whereas the single-hop system has the worst performance. In addition, it was shown that increasing the impulsive noise probability or the channel variance will considerably degrade performance.

\section{ACKNOWLEDGMENT}

This research has been carried out within the "Smart InBuilding Micro Grid for Energy Management" project funded by EPSRC (EP/M506758/1) and supported by Innovate UK (Innovate UK Project 101836).

\section{REFERENCES}

[1] M. Zimmermann and K. Dostert, "A multipath model for the powerline channel," IEEE Trans. Commun., vol. 50, pp. 553-559, Apr. 2002.

[2] M. Rozman, A. Ikpehai, B. Adebisi, and K. M. Rabie, "Channel characterisation of cooperative relaying power line communication systems," in Proc. IEEE Int. Symp. Commun. Systems, Networks and Digital Signal Processing (CSNDSP), pp. 1-5, Jul. 2016.

[3] A. Ikpehai, B. Adebisi, and K. M. Rabie, "Broadband PLC for clustered advanced metering infrastructure (AMI) architecture," Energies, vol. 9, pp. 1-19, Jul. 2016.

[4] A. Ikpehai, B. Adebisi, K. M. Rabie, R. Haggar, and M. Baker, "Experimental study of 6LoPLC for home energy management systems," Energies, vol. 9, no. 12, p. 1046, 2016.

[5] L. Lampe and A. Vinck, "Cooperative multihop power line communications," in Proc. IEEE Int. Symp. Power Line Commun. and Its Appl. (ISPLC), pp. 1-6, Mar. 2012.

[6] A. Tonello, F. Versolatto, and S. D'Alessandro, "Opportunistic relaying in in-home PLC networks," in Proc. IEEE Global Commun. Conf. (GLOBECOM), pp. 1-5, Dec. 2010.

[7] X. Cheng, R. Cao, and L. Yang, "Relay-aided amplify-and-forward powerline communications," IEEE Trans. Smart Grid, vol. 4, pp. 265 272, Mar. 2013

[8] S. D'Alessandro and A. M. Tonello, "On rate improvements and power saving with opportunistic relaying in home power line networks," EURASIP J. Advances Signal Process., vol. 2012, pp. 1-17, Sept. 2012.

[9] W. Bakkali, P. Pagani, and T. Chonavel, "Energy efficiency performance of relay-assisted power-line communication networks," in Proc. IEEE Consumer Commun. and Networking Conf. (CCNC), pp. 525-530, Jan. 2015.

[10] W. Bakkali, P. Pagani, T. Chonavel, and A. M. Tonello, "Energy efficiency performance of decode and forward MIMO relay PLC systems," in Proc. IEEE Int. Symp. Power Line Commun. and Its Appl. (ISPLC), pp. 225-230, Mar. 2016 (accepted).

[11] K. M. Rabie, B. Adebisi, and A. Salem, "Improving energy efficiency in dual-hop cooperative PLC relaying systems," in Proc. IEEE Int. Symp. Power Line Commun. and Its Appl. (ISPLC), pp. 196-200, Mar. 2016.

[12] K. M. Rabie, B. Adebisi, A. M. Tonello, and G. Nauryzbayev, "For more energy efficient dual-hop DF relaying power line communication systems," IEEE Systems Journal, Jan. 2017.

[13] K. M. Rabie, E. Alsusa, A. D. Familua, and L. Cheng, "Constant envelope OFDM transmission over impulsive noise power-line communication channels," in Proc. IEEE Int. Symp. Power Line Commun. and Its Appl. (ISPLC), pp. 13-18, Mar. 2015.

[14] F. H. Juwono, Q. Guo, Y. Chen, L. Xu, D. D. Huang, and K. P. Wong, "Linear combining of nonlinear preprocessors for OFDM-based powerline communications," IEEE Trans. Smart Grid, vol. 7, pp. 253-260, Jan. 2016.

[15] K. M. Rabie and E. Alsusa, "Performance analysis of adaptive hybrid nonlinear preprocessors for impulsive noise mitigation over power-line channels," in Proc. IEEE Int. Conf. Commun. (ICC), pp. 728-733, Jun. 2015. 
[16] W. Bakkali, M. Tlich, P. Pagani, and T. Chonavel, "A measurementbased model of energy consumption for PLC modems," in Proc. IEEE Int. Symp. Power Line Commun. and Its Appl. (ISPLC), pp. 42-46, Mar. 2014.

[17] M. Ghosh, "Analysis of the effect of impulse noise on multicarrier and single carrier QAM systems," IEEE Trans. Commun., vol. 44, pp. 145147, Feb. 1996.

[18] A. Dubey and R. K. Mallik, "PLC system performance with AF relaying," IEEE Trans. Commun., vol. 63, pp. 2337-2345, Jun. 2015.

[19] A. Dubey, R. K. Mallik, and R. Schober, "Performance analysis of a multi-hop power line communication system over log-normal fading in presence of impulsive noise," IET Commun., vol. 9, no. 1, pp. 1-9, 2015.

[20] K. M. Rabie, B. Adebisi, and M. Rozman, "Outage probability analysis of WPT systems with multiple-antenna access point," in Proc. IEEE Int. Symp. Commun. Systems, Networks and Digital Signal Processing (CSNDSP), pp. 1-5, Jul. 2016.

[21] M. T. Kakitani, G. Brante, R. D. Souza, and A. Munaretto, "Comparing the energy efficiency of single-hop, multi-hop and incremental decodeand-forward in multi-relay wireless sensor networks," in Proc. IEEE Int Symp Personal, Indoor and Mobile Radio Commun. (PIMRC), pp. 970974, Sept. 2011. 